\title{
Review of: "Proteomic phenotyping of stimulated Mueller cells uncovers profound pro-inflammatory signaling and antigen-presenting capacity"
}

Luigi Donato ${ }^{1}$

1 University of Messina

Potential competing interests: The author(s) declared that no potential competing interests exist.

Schmalen et al. wrote a very interesting article describing how "Proteomic phenotyping of stimulated Mueller cells uncovers profound pro-inflammatory signaling and antigen-presenting capacity". The manuscript represents an interesting way to discover new scenarios for retinal degenerations. I suggest only several minor revisions needed to update and improve the reliability of the paper:

- The whole manuscript could be improved with more recent literature about retinal cell death and related biochemical pathways such as oxidative stress and neurotransmission. Regarding these, I suggest to add the following references to manuscript PMID: 34440511 , PMID:34058230 and PMID: 33801777.

- Finally, manuscript requires English revisions and typos correction. 